\title{
Nous autres / vous autres / eux autres, pronoms catégoriels
}

\author{
Hilgert Emilia \\ Université de Reims Champagne-Ardenne \\ CIRLEP EA 4299 \\ emilia.hilgert@univ-reims.fr
}

\section{Introduction}

Cette étude ${ }^{1}$ est consacrée aux pronoms nous autres, vous autres et eux autres, peu décrits et présentés sous une étiquette qui ne correspond que partiellement à leur emploi et à leur signification. En effet, ils sont mentionnés comme ayant deux emplois ( $c f$. Le Bon usage, GMF $4^{\mathrm{e}}$ édition) : (i) en tant que déictiques conjoints au verbe et dépourvus de sens oppositif, usage correspondant au français du Québec et à certains parlers régionaux de Belgique ou de l'Ouest, et (ii) en tant que pronoms disjoints employés à des fins contrastives en français normé. Sur ces derniers, le Dictionnaire historique de la langue française (2006) informe que, par rapport aux emplois de nous comme pronom personnel qui sont fixés avant la fin $\mathrm{du} \mathrm{XII}^{\mathrm{e}}$ s., l'association à autres, en emploi renforcé marquant une forte distinction, ne date que du $\mathrm{XVI}^{\mathrm{e}}$ siècle. C'est ce deuxième emploi que nous souhaitons discuter, celui du français standard, c'est-à-dire l'emploi disjoint ou accentué qu'illustrent les exemples suivants :

1) Nous autres femmes, nous avons un sens que les hommes ne possèdent pas. (Mauriac C., La Marquise sortit à cinq heures, 1961, p. 63)

2) La grande supériorité de vous autres dans l'enseignement sur nous autres dans les chemins de fer, c'est que lorsque vous vous salissez les mains, c'est avec du blanc de tableau, tandis que nous autres, c'est avec du noir de charbon! (L'Hôte J., Le Mécréant ou les preuves de l'existence de Dieu, 1981, p. 29-30)

3) Tu cries, mais tu ne dis pas ce que tu penses. "Oh! Moi, bien sûr, dit André, moi je crierais ce qu'on voudra, mais eux autres c'est pas pareil : ils sont alsaciens; ils ont des devoirs envers la France. » (Sartre J.- P., La Mort dans l'âme, 1949, pp. 260-261)

La problématique de ces pronoms est stimulante pour plusieurs raisons. D'abord, les quelques mentions des dictionnaires et des grammaires du français font part de l'interprétation contrastive de ces pronoms, qui intrigue par le fait que nous / vous / eux accentués, utilisés sans l'ajout de autres, peuvent aussi produire un effet contrastif (nous, nous le savons laisse entendre que d'autres ne le savent pas), comme le font tous les pronoms toniques, caractérisés par leur trait ostensif, mis en lumière par Kleiber (1994 : 135 $139^{2}$ ). On peut donc se demander ce qui distingue l'emploi contrastif de nous de l'emploi contrastif de nous autres. Il faudrait aussi préciser la terminologie et les critères d'analyse utilisés, parce que, si certains spécialistes les appellent "pronoms renforcés », ils proposent une description syntaxique due à l'ajout de autres, alors que ceux qui parlent de "pronoms contrastifs » prennent en compte un critère sémantique $^{3}$. Enfin, les rares mentions des pronoms contrastifs sont consacrées à nous autres et vous autres, en passant sous silence l'emploi de eux autres disjoint, qui, pour rare qu'il soit ${ }^{4}$, complète le paradigme de ces pronoms ${ }^{5}$.

Notre argumentation tentera de montrer, sur la base d'exemples de Frantext, que nous autres, vous autres et eux autres fonctionnent comme des 'pronoms catégoriels' qui encodent la double idée d'identité catégorielle et d'altérité associée à cette identité, l'idée d'opposition ou de contraste, qui ne s'active pas 
systématiquement, n'étant qu'une conséquence de l'affirmation de l'identité - altérité. Notre analyse des pronoms disjoints pluriels avec autres (désormais Pron.+autres) s'opposera donc partiellement à celle proposée par Rosier (2006), qui trouve que nous autres et vous autres créent une interprétation partitive : en s'appuyant sur la valeur 'confrontative' de ces pronoms, l'auteure considère que l'affirmation de la différence déclenche un mécanisme de partition qu'elle appelle 'partition inverse' et qu'elle explique par le fait que la référence à l'ensemble désigné par nous autres supposerait «obligatoirement un ensemble plus vaste qui le contient » (p. 164). Ou, comme le dit l'auteure, on partirait « de la partie pour remonter vers le tout (nous autres + vous autres = tout) » (p. 163). Ou, en d'autres termes, il y aurait « l'affirmation d'une identité (nous) contrastive (autres), qui renvoie à un ensemble supérieur (les autres que nous) » (p. 162). Mais les exemples donnés par Rosier ont de quoi surprendre : nous autres Espagnols renverrait à un 'ensemble supérieur' ceux qui ne sont pas espagnols. Or il n'est pas logique de considérer, nous semble-til, que ce dernier ensemble, ceux qui ne sont pas espagnols, plutôt complémentaire, soit en même temps « un ensemble supérieur» dont fait partie l'ensemble nous autres Espagnols, conformément à la formule «nous autres+vous autres = tout». On ne sait pas si, dans l'opinion de Rosier, l'ensemble supérieur contenant nous autres Espagnols peut être réellement ceux qui ne sont pas espagnols ou un autre 'tout', si l'ensemble supérieur à nous autres femmes opposé à vous autres hommes est ce dernier ou un autre 'tout'. On serait tenté de penser que l'ensemble supérieur est, dans tous les cas, celui des humains. Mais cela ne se vérifie pas toujours: s'il y a un contraste affirmé entre nous autres cheminots et vous autres enseignants, ou entre nous autres ouvriers et vous autres patrons, les deux classes respectivement opposées ne forment pas l'ensemble des humains. La piste de la partition inverse ne nous semble pas valide.

Nous proposerons une analyse qui prend en compte l'effet contrastif des Pron.+autres, en avançant l'hypothèse que ces pronoms expriment un contraste catégoriel, différent du contraste produit par les pronoms simples en emploi accentué. Nous développerons notre point de vue en trois volets : premièrement, nous décrirons brièvement ces pronoms catégoriels, pour montrer qu'il existe plusieurs constructions distinctes, que nous ne tenterons pas de réduire à une forme canonique mais que nous présenterons dans une perspective de grammaire de constructions; deuxièmement, nous ferons quelques remarques sur le mécanisme énonciatif à l'œuvre dans l'emploi de Pron.+autres et sur l'apport de autres et de l'apposition identitaire dans cet assemblage, qui nous permettront d'opposer l'identité catégorielle explicite qu'ils signifient à une identité de groupe contextuellement délimité ; enfin, l'observation du fonctionnement de ces pronoms au niveau de la phrase en prenant en compte la prédication nous mènera en plein domaine pragmatique. Nous insisterons sur un aspect original de ces pronoms, à savoir l'association de la deixis et de la catégorisation ou l'association de l'énonciation et de la généricité au niveau des énoncés. C'est de l'observation de cette double propriété que l'on tirera le plus de profit dans l'explication de ces pronoms, avec une ouverture vers un volet pragmatique de leurs emplois, consistant dans la réalisation de différents actes de langage indirects.

\section{Description}

\subsection{Pronoms disjoints du verbe}

Comme nous l'avons déjà dit, la première des propriétés des pronoms catégoriels nous autres / vous autres / eux autres est leur position disjointe du verbe et, donc, leur emploi accentué, marqué par la ponctuation et par la prosodie :

4) Nous autres, hommes, nous devons considérer le phénomène de la vie et de la mort comme inéluctable. Lorsqu'on comprend, on accepte. (Berr H., Journal 1942-1944, 2008, p. 239)

5) Je sais que nous n'en aurons jamais fini avec eux. Ils nous accompagnent où que nous allions, formant une immense chaîne qui les relie à nous autres, les rescapés. (Veil S., Une vie, 2007, p. 332) 
6) Vous autres les femmes, vous aimez que les feignants! (Simonin A., Du mouron pour les petits oiseaux, 1960, p. 13)

7) Toutes les choses que je pense, vous les pensez sûrement aussi, vous autres, les vertueux, seulement vous n'y comprenez rien. Vous êtes pleins de complaisance pour vous-mêmes... (Duhamel G., Chronique des Pasquier. 6. Les Maîtres, 1937, p. 164)

8) A-t-on idée de courir les routes par une chaleur pareille! Tout cela pour un sanglier! Et votre Alexis qui déserte à cause d'un chenil ! Vous autres, nobles, la chasse vous perdra! (Chandernagor F., L'Enfant des Lumières, 1995, p. 302)

9) Bloch nous quitta devant sa porte, débordant d'amertume contre Saint-Loup, lui disant qu'eux autres, "beaux fils " galonnés, paradant dans les états-majors, ne risquaient rien, et que lui, simple soldat de $2^{e}$ classe, n'avait pas envie de se faire "trouer la peau pour Guillaume ». (Proust M., Le Temps retrouvé, 1922, p. 740)

10) Nous seuls avons le droit d'être à l'image et à la ressemblance, à l'imitation de Jésus ; de souffrir à l'image et à la ressemblance; à l'imitation de Jésus. Eux autres, les malheureux, ils n'ont pas même le droit d'être en croix. (Péguy C., Le Mystère de la charité de Jeanne d'Arc, 1910 , p. 65)

La deuxième propriété des Pron.+autres disjoints est la limitation aux trois personnes du pluriel, (excepté les créations de Sartre, qui s'adresse dans sa correspondance au «Castor» avec la formule "vous autre $»^{6}$ ).

\subsection{Différentes positions dans la phrase}

Les pronoms catégoriels (Pron.+autres), la plupart du temps coréférentiels avec un pronom conjoint de même personne, fonctionnent généralement comme des constructions détachées, mais, malgré une certaine idée reçue, ils ne sont pas réservés au doublement du sujet, ni à la position topicalisée. Ils apparaissent :

(i) en construction détachée topicalisée ou non, coréférentielle avec un pronom conjoint sujet (parfois on à la place de nous) :

- nous autres, hommes, nous devons considérer le phénomène de la vie et de la mort comme inéluctable ;

- nous savons nous autres, Parisiens, que P;

- des fois dans notre métier, nous autres, on trouve les truands plus sympathiques que les plaignants;

- toutes les choses que je pense, vous les pensez sûrement aussi, vous autres, les vertueux;

- eux autres, les malheureux, ils n'ont pas même le droit d'être en croix;

(ii) dans une construction sous-entendant le sujet, sans reprise par un pronom conjoint (l'absence de pronom conjoint s'observe aussi dans des exemples du type ma femme et moi-même vous souhaitons de joyeuses fêtes) :

- nous autres, Français, sommes divers à tous égards;

- vous avez devant vous une belle incarnation de ce que vous autres, philosophes, appelez, je crois, un couple dialectique

- Bloch nous quitta devant sa porte, [...] disant qu'eux autres, "beaux fils " galonnés, paradant dans les états-majors, ne risquaient rien;

(iii) en construction détachée, coréférentielle avec un pronom complément d'objet direct :

- ce "vingtième » qui vient de tripler, cette dîme qui nous écorche, toutes ces taxes enfin qui ne cessent de croître et nous écrasent autant que les paysans, nous autres " privilégiés ";

- vous autres, nobles, la chasse vous perdra; 
(iv) dans un syntagme prépositionnel (SP) détaché coréférentiel avec un pronom complément d'objet indirect ou dans un SP c.o.i. non détaché :

- qui nous font tant de mal, à nous autres, les désaxés ;

- [ils sont] reliés à nous autres, les rescapés;

(v) dans un SP topicalisé : pour nous autres, enfants, $P$.

\subsection{Désignation identitaire ou identité inférable}

Une propriété essentielle des pronoms nous autres / vous autres / eux autres, insuffisamment mise en évidence, est le fait qu'ils sont généralement accompagnés d'une expression désignant une identité de classe :

11) Mais voyez-vous, nous avons, nous autres Florentins, découvert le remède de ce chancre purulent : l'or. Contre les plaies morales de l'humanité, la panacée, c'est la richesse. (Tournier M., Gilles et Jeanne, 1983, p. 71)

12) C'est bizarre, nous autres, pupilles de l'Assistance, on espère toujours... On n'a pas l'amour exclusif. Les parents d'occasion, on s'en accommode... (Szczupak-Thomas Y., Un diamant brut Vézelay-Paris 1938-1950, 2008, p. 106)

13) Si nous n'existions pas, nous autres, les mauvais payeurs, nous autres, les pauvres gens, vous claqueriez du bec, vous et votre séquelle ; vous n'auriez rien à faire au monde. (Duhamel G., Chronique des Pasquier. 2. Le Jardin des bêtes sauvages, 1934, p. 164)

14) [...] je ne suis pas obligée, comme vous autres les chanteurs, de mener une vie régulière : tant d'heures de sommeil, des repas à heures fixes, et caetera! (Benjamin L., L'Opéra du fond des mers, 1983, p. 33)

15) Il y aurait... bataille, répéta machinalement Couche-tout-nu. - À moins que les Loups n'aient peur des Dévorants ! ajouta Morok. [...] - Pourquoi tant de misère pour nous et tant de bonheur pour eux? - Ils ont dit que les carriers étaient des bêtes brutes, bonnes à monter dans les roues de carrière comme des chiens de tournebroche, dit un émissaire du baron Tripeaud. - Et qu'eux autres Dévorants se feraient des casquettes avec la peau des Loups, ajouta un autre. (Sue E., Le Juif errant, 1845, p. 674)

16) Nous autres, qui vivons entre l'Atlantique et l'Oural; nous autres qui sommes l'Europe, disposant avec l'Amérique, sa fille, des sources et des ressources principales de la civilisation; nous autres, qui avons de quoi manger, nous vêtir, nous loger, nous chauffer [...], que ne dressons-nous, tous ensemble, la fraternelle organisation qui prêtera son concours aux autres? (De Gaulle C., Discours et messages. 3. Avec le renouveau. 1958-1962, 1970, p. 86)

17) Voyant rouge si un camarade regardait de travers sa chevelure, dégainant l'épée ou le poignard chaque fois qu'un homme disait : "Vous autres, de Toulouse ... », se cassant la tête pour deviner si telle dame l'avait bien regardé d'une certaine façon ... (Oldenbourg Z., Les Cités charnelles ou l'histoire de Roger de Montbrun, 1961, p. 19)

L'expression identitaire qui accompagne les pronoms contrastifs prend différentes formes : celle du nom générique sans déterminant, simple ou modifié, séparé ou non du pronom par une virgule, celle du syntagme nominal toujours défini de forme les $N$ (générique également) avec modifieur ou non, détaché ou non, celle d'une proposition relative, enfin, celle d'un syntagme prépositionnel détaché ou non ( $c f$. vous autres, de Toulouse; nous autres dans les chemins de fer). Il est difficile de dire s'il y a une construction prototypique et d'autres qui gravitent autour de celle-ci ou s'il y en une plus représentative que les autres ${ }^{7}$. Nous préférons les considérer comme des réalisations syntaxiques différentes, mais très voisines par le fait qu'elles assurent toutes, quelle que soit leur forme, l'expression de l'identité catégorielle de la classe à laquelle appartient l'énonciateur de «nous autres » ou de la classe à laquelle appartient la personne à laquelle il adresse la formule «vous autres », ou encore de la classe pointée par 
«eux autres ». Lorsque les Pron.+autres sont suivis d'adjectifs non substantivés, ces adjectifs sont toujours en construction détachée et ne servent plus à catégoriser ou à indiquer une identité, mais à exprimer une ou plusieurs propriétés de la classe implicite, inférable du contexte, à laquelle renvoie nous autres, comme dans :

18) Leur beau parler amplifiait la distance entre nous autres, laids, humbles, serviles, et ces Seigneurs. (Szczupak-Thomas Y., Un diamant brut. Vézelay-Paris 1938-1950, 2008, p. 164)

L'absence d'apposition identitaire ou de nom générique d'identité n'empêche pas l'inférence de l'identité de Pron.+autres. En effet, l'absence de ces éléments est comblée par l'inférence de l'identité grâce au contenu propositionnel ou à une relative :

19) ... cette Cheviré qui passait son temps à la pêche, au lieu de tenir sa maison elle-même, de s'user les mains dans la lessive et de supporter ses enfants, comme nous autres. (Dormann G., La Petite main, 1993, p. 61-62)

20) Dieu est Dieu parce qu'il a le pouvoir de ressentir comme des souffrances d'amour ce que nous autres appelons des souffrances d'amour-propre. (Huguenin J.-R., Journal, 1993, p. 242-243)

21) Moi aussi ça me faisait de l'effet. J'ai pas touché aux sirops, ni même aux petits fours qu'étaient offerts alentour par les gens du monde... Ils ont l'habitude eux autres de mélanger la boustifaille avec les émotions magiques... Tout leur est bon les sagouins ! Pourvu qu'ils avalent... Ils peuvent jamais s'interrompre. (Céline L.-F., Mort à crédit, 1936, p. 583)

Dans (19), l'identité de nous autres est comprise comme « nous qui tenons notre maison nous-mêmes, c'est-à-dire les bonnes mères de famille. Dans (20), nous autres est assimilé aux « humains » grâce à «l'amour-propre» et à l'opposition Dieu. Dans (21), eux autres reprend anaphoriquement les gens du monde. De même, dans (2) : la grande supériorité de vous autres dans l'enseignement sur nous autres dans les chemins de fer, les SP à signification distinctive dans l'enseignement et dans les chemins de fer laissent inférer les enseignants vs les cheminots. Enfin, dans les emplois des Pron.+autres sans apposition, l'identité catégorielle de leurs référents est comprise par inférence du contexte discursif :

22) Il a traversé lentement, même quand les tirs ont repris. Se tourne vers Boris tout seul : - À toi! On peut avoir besoin de ta grenade. Vous autres, vous attendez !! Les autres, les bienheureux F.F.I. qui se recroquevillent derrière le char... (Schreiber B., Un silence d'environ une demiheure, 1996, p. 953-954)

23) Pendant les interrogatoires, je lui sentais moins de cœur à l'ouvrage. Il range ses menottes en poche. - Tu sais, des fois dans notre métier, nous autres, on trouve les truands plus sympathiques que les plaignants. (Boudard A., Les Enfants de chœur, 1982, p. 34-35)

Dans l'énoncé (22), vous autres renvoie à un groupe contextuellement déterminé dans la situation d'énonciation par le geste d'ostension ou le regard supposés accompagner l'énonciation, mais le contenu propositionnel donne aussi l'identité catégorielle de ce groupe : il s'agit des bienheureux F.F.I. derrière le char. L'énoncé (23) permet l'inférence d'un corps de métier, à savoir (nous autres) les policiers.

On peut représenter de manière schématique ces formes complexes et assez hétérogènes par «nous autres / vous autres / eux autres $I D$ », ID signifiant l'apposition identitaire explicite ou l'identité inférable à partir du contenu propositionnel ou de la situation de communication :

- nous autres $N$ (nous autres Florentins)

- nous autres, $N$, (nous autres, hommes,)

- nous autres, $N$ exp., (nous autres, pupilles de l'Assistance,)

- nous autres, les $N$, (nous autres, les hommes,)

- nous autres, Adj., (nous autres, Ø [inférence] laids, humbles, serviles)

- nous autres, $P$ Rel (nous autres, qui ...)

- nous autres, SP (nous autres, de Toulouse)

- nous autres $\varnothing$ [inférence] 


\section{Interprétation}

\subsection{Sens et référence de nous autres / vous autres / eux autres}

Il est difficile de gloser le sens des Pron.+autres sans être amené à faire appel en même temps à l'identité explicite ou inférable qui les accompagne et à leur emploi accentué. Commençons par ce dernier. Comme l'explique Kleiber (1986 et 1994: 136), la position accentuée des pronoms, en l'occurrence leur détachement syntaxique, peut s'assimiler au geste. Il en résulte le trait déictique propre aux pronoms toniques, qui n'est pas seulement celui de la référence donnée par la situation de discours comme pour je et $t u$, mais qui provient de l'effet indexical de la position accentuée, qui provoque «l'isolement, l'identification autonome du référent » rendu saillant. Autrement dit, dans le cas des pronoms accentués, l'accent est d'une part un signal indexical (qui sert à l'identification des référents, à la manière d'un geste) et assure, d'autre part, une indépendance référentielle à leurs référents (raison pour laquelle les pronoms accentués sont appelés « ontiques » par Moignet, $1981^{8}$ ). Nous / vous / eux accentués, utilisés avec ou sans autres, attirent donc l'attention sur des pluralités dont l'existence ne dépend pas d'une prédication.

Le sens de ces pronoms est bien connu. Nous inclut nécessairement le je du locuteur et peut être inclusif, s'il inclut l'allocutaire ou exclusif, s'il l'exclut, en incluant alors des personnes in absentia ou in praesentia, mais n'ayant pas le statut d'interlocuteur ( $c f$. Kerbrat-Orrechioni, 1999 : 46-47). Le pronom disjoint correspond à l'idée de groupe incluant $\{m o i+t o i\},\{m o i+v o u s\},\{m o i+v o u s+e u x\}$ ou $\{m o i+$ $e u x\}$ correspondant aux pronoms accentués. Il en résulte deux sens de nous accentué contrastif :

(i) nous (autres) inclusif $=\{m o i+t o i\}$ ou $\{m o i+v o u s\}$, par exemple, dans un dialogue entre membres d'une même classe socioprofessionnelle ( $c f$. nous autres [enseignants] nous savons que certaines réformes troublent les élèves) ; cette glose présuppose une idée de catégorie ou classe d'appartenance, dans le sens où le locuteur individuel qui dit nous autres, membre d'une classe professionnelle, ne peut utiliser le nous autres inclusif que si son ou ses allocutaires appartiennent à la même catégorie socioprofessionnelle ;

(ii) nous (autres) exclusif $=\{m o i+e u x$ générique $\}$ ou $\{m o i+e u x$ (in praesentia, faisant partie de la même classe que le locuteur) $\}$ ou $\{m o i+$ eux générique $\}$; ce dernier, c'est le cas le plus fréquent, celui du renvoi à la classe à laquelle se sent appartenir le locuteur; la classe d'appartenance est désignée par l'apposition ID, sous la forme d'un SN générique les $N$ (nous autres, les Parisiens / les hommes) ou du $N$ générique sans déterminant, qui renvoie à la classe d'appartenance du locuteur par le double jeu de l'intension sémantique et de l'extension référentielle (nous autres Florentins, nous autres, hommes).

Avec vous (autres), toujours collectif (et pas 'de politesse') et dépendant uniquement de la situation de l'énonciation (qu'elle soit orale ou écrite) :

(i) les allocutaires constituent un groupe contextuellement déterminé, montré du regard ou montré par un geste, vous (autres) renvoyant simultanément à plusieurs allocutaires, auquel cas vous $=$ toi + toi + toi, comme dans Vous autres [les F.F.I.], vous attendez !

(ii) vous (autres) renvoie à un ou plusieurs allocutaires et, simultanément, à une classe à laquelle ils appartiennent, sous-entendant donc toi ou vous sg. / pl $_{\text {. }}+$ eux générique (comme dans vous autres, nobles, la chasse vous perdra).

Eux (autres) a le sens d'une pluralité d'individus qui ne participent pas à l'acte d'énonciation, dont le locuteur fait mention in absentia. Cette forme accentuée signifie un groupe défini, auquel l'indication identitaire ou son inférence confèrent le trait [+humain] et un statut catégoriel, générique, s'entendant comme une classe.

Les pronoms nous autres ID / vous autres ID expriment ainsi l'association (insolite, inédite) de deux processus a priori difficilement associables : l'énonciation et la catégorisation. L'ancrage énonciatif de 
ces pronoms pluriels est donné par leurs composantes 'd'énonciation' moi / toi / vous, dont le sens instructionnel, procédural, indique comment trouver le locuteur et l'interlocuteur'. L'ancrage catégoriel, lui, est annoncé par autres et est explicitement donné par l'expression identitaire, qui entretient avec cet adjectif une relation anaphorique. Autrement dit, si ID n'est pas explicite, autres signale de toute manière qu'il s'agit aussi de eux de classe et qu'il ouvre la voie à une catégorisation qu'il faudra trouver par des moyens anaphoriques.

\subsection{Sens et référence de ID}

La composante ID de ces pronoms ne désigne pas l'identité 'civile', c'est-à-dire telle qu'elle est donnée à un individu par le nom propre, qui assure dénominativement son identification, sans le décrire. Elle exprime, au contraire, une identité de classe, de communauté, de collectivité ou de groupe, qui découle du $\mathrm{N}$ ou du SN générique en apposition ou inférable. Et pourtant, l'association des pronoms personnels pluriels accentués avec une apposition identitaire catégorielle va de pair avec le fait que, référentiellement, les pronoms personnels ont la propriété d'indiquer, même seuls, une identité autre que celle correspondant simplement au rôle interlocutif ' $j e=$ celui qui parle' et ' $t u$ = celui à qui je parle'. Kleiber (2012) rappelle à cet égard les remarques de Bonnard (1950) et de Tamba (1994) :

- le premier identifie, comme première fonction des trois pronoms personnels, celle d'indiquer l'identité : «Les pronoms personnels désignent des personnes ou des choses; ils comportent une indication d'identité : moi, toi, lui »(Bonnard, 1950 : 143, in Kleiber, 2012);

- la deuxième remarque la possibilité pour les pronoms de $1^{\text {ère }}$ et $2^{\mathrm{e}}$ personnes d'être accompagnés d'appositions "identificatoires », comme dans les exemples suivants : Je, soussigné X, atteste que ... ; Toi, François, si tu continues, tu vas prendre une de ces raclées! (Tamba, 1994 : 223, in Kleiber, 2012 : $150 \mathrm{ss}$, qui souligne que « ces appositions identificatoires ne pourraient exister si les pronoms je et $t u$ ne donnaient pas lieu à une identification autre que celle du rôle interlocutif indiqué. »).

L'apposition identitaire des Pron.+autres ne s'accorde pourtant que partiellement avec l'identité que supposent le moi et toi inclus dans les pluralités visées, parce que l'identité n'est pas celle de l'individu qui parle et qui serait équivalente à son nom propre : la composante ID efface les identités individuelles au profit de l'identité de classe d'appartenance de moi et de toi. A un degré intermédiaire, Nous autres, les Sanchez (titre de roman qui peut être interprété comme un calque sur l'espagnol) désigne une famille qui a une identité civile, donnée par le nom de famille, mais qui assume aussi une identité provenant de ses particularités de famille mixte, différente de celles du milieu où elle vit ${ }^{10}$.

L’identité de Pron.+autres ID est donnée par le nom ou le SN génériques de l'apposition identitaire et est interprétable comme une somme de propriétés dénotatives et connotatives, ces dernières étant diverses, positives ou négatives, gravitant autour de jugements stéréotypés (les cheminots se salissent les mains avec du noir de charbon, les policiers sont censés ne pas avoir de sympathie pour les truands, etc.). Or, l'identité catégorielle est censée se retrouver chez chacun des individus de la classe. L'identité individuelle de type catégoriel découle donc de celle de la classe d'appartenance : le je qui prononce nous autres femmes est nécessairement une femme. Il y a une stratégie communicative dans l'utilisation du pronom nous autres, qui consiste dans le fait qu'en se servant des pronoms contrastifs l'énonciateur met en avant l'idée d'appartenance à une classe et non l'affirmation de l'individualité, pour faire passer ses idées sous couvert d'autorité générique. Comme on l'a déjà dit, nous autres / vous autres sont biréférentiels et réunissent énonciation et généricité, alors que eux autres signifie que le moi / je locuteur vise et catégorise une classe à laquelle lui et les siens n’appartiennent pas.

Enfin, une remarque sur la référence des Pron.+autres ID, qui prend en compte aussi bien le processus sémantico-référentiel de leur composante pronominale nous / vous / eux que l'interprétation que donne leur composante ID. Ces formes de pluriel impliquent la référence à des ensembles animés ou, plus précisément, et d'une manière très particulière, à des classes ou des catégories d'individus qui ne peuvent être conçus que comme ayant le trait fondamental [+humain] (cf. nous autres, hommes) auquel peuvent s'ajouter d'autres traits catégoriels différenciateurs, par exemple [+éditeur], [+parent], etc. : 
24) De toute façon, nous autres les parents, malgré les contacts directs, malgré les conseils élus sur listes concurrentes par une minorité d'électeurs, malgré les réunions avec les enseignants, nous n'arrivons guère à nous débarrasser à leur égard d'un sentiment analogue à celui que nous inspirent ces corporations incontrôlables qui s'occupent de mécanique, de plomberie, d'électricité ... (Bazin H., L'école des pères, 1991, p. 99)

25) Vous ne l'avez même pas lu, n'est-ce pas ? J'avais mis les pages 36, 123 et 247 à l'envers, elles y sont toujours. - Classique... Dire que nous autres, les éditeurs, si futés que nous soyons, nous nous laissons encore prendre à ça! Que répondre, Benjamin? Que répondre à ce mec? (Pennac D., La Petite marchande de prose, 1989, p. 20)

Il y a, certes, des emplois des Pron.+autres suivis d'un nom [-humain] ou d'un groupe nominal comportant un tel nom, comme dans :

26) Nous autres, civilisations, nous savons maintenant que nous sommes mortelles. (Valéry P., Variété I, 1924)

27) En 1952, Antoine Pinay, alors président du Conseil et ministre des Finances, décréta une baisse des prix, de $5 \%$ je crois, que nous autres, avant-garde éclairée du prolétariat, considérions comme une scandaleuse machination de la bourgeoisie impérialiste. (Genette G., Bardadrac, 2006, p. 360)

28) Quand vous autres, les partis, vous exigez que les gouvernements procèdent de vous-mêmes, en quoi cela consiste-t-il? En ceci que vous juxtaposez dans les conseils des ministres sans cohésion et sans chef, vos rivalités, vos oppositions réciproques, vos ambitions, vos impuissances. (De Gaulle C., Discours et messages. 2. Dans l'attente. 1946-1958, 1970, p. 348)

Or, il n'y a aucune contradiction entre le trait général [+humain] que supposent les Pron.+autres et des noms comme parti et prolétariat (et donc avant-garde du prolétariat) : ces derniers sont des noms collectifs définis comme des 'regroupements' d'individus ayant le trait [+humain] (cf. Lammert, 2010). Le nom civilisation est utilisé de manière stylistique et renvoie, par des mécanismes métonymiques, et grâce au verbe savoir, aux humains qui constituent les civilisations.

Il n'est pas inutile de souligner le trait [+humain] des pronoms catégoriels : si cela est une évidence pour nous autres ou vous autres, grâce aux pronoms d'énonciation nous / vous qu'ils contiennent, le trait [+humain] doit être souligné pour eux autres, dont le pronom 'tête' eux n'est a priori pas une marque de l'énonciation: eux autres ne se comprend pas comme pouvant référer à un ensemble de livres, par exemple. Il acquiert le trait [+humain] du paradigme.

\subsection{Apport sémantico-syntaxique de autres}

Il convient de s'interroger sur l'apport sémantique de autres, surtout parce que les pronoms nous, vous et eux peuvent fonctionner sans autres, tout en étant accompagnés d'une apposition explicitant l'identité de la classe d'appartenance (nous / vous / eux $I D^{11}$ ) :

29) La salle de restaurant luxueuse, d'un côté, le groupe du voyage organisé, nous, les péquenots, de l'autre, les clients normaux, cette fille bronzée, avec son père, chic. Elle mangeait ce que j'ai su plus tard être un yaourt. (Ernaux A., Se perdre, 2001, p. 141)

30) Nous, les communistes, nous savons ce qu'est la dialectique, c'est pourquoi nous comprenons le dessous des apparences. (Schreiber B., Un silence d'environ une demi-heure, 1996, p. 214)

31) Vous, les Européens, vous êtes malades de théorie mais la guerre n'est pas une question théorique. (Debray R., Loués soient nos seigneurs : une éducation politique, 1996, p. 184)

32) Nous, hommes politiques africains, devons avoir des idées très claires sur la situation de notre peuple. (Fanon F., Les damnés de la terre, 1961, p. 236) 
33) Toluy ne le comprit pas. Il avait peine à imaginer que l'on puisse faire la guerre à cause d'un simple désaccord religieux. Eux, les Mongols, se battaient pour acquérir de nouveaux herbages, de nouveaux fleuves et y faire croître et abreuver toujours plus de chèvres et de juments. (Lanzmann J., La Horde d'or, 1994, p. 336)

L'apposition du type les $N$ générique ou $N$ générique modifié sans déterminant désigne l'identité de la classe mise en évidence par les pronoms accentués nous / vous / eux. Elle produit un contraste identitaire catégoriel. Si l'apposition est supprimée, le contraste n'est plus de type catégoriel, mais de groupe spécifique, c'est-à-dire non seulement qu'il est donné par la prédication (nous, nous savons ce qu'est la dialectique transmet indirectement le message suivant : vous ne savez pas ce qu'est la dialectique), mais que, surtout, l'énoncé peut concerner n'importe quel groupe qui s'auto-désigne par nous, sans aucune idée de classe d'appartenance. Le contraste catégoriel entre deux classes peut être explicite, comme dans nous, les péquenots vs les clients normaux, élégants ou implicite, comme dans vous, les Européens, vous êtes malades de théorie qui laisse entendre que le locuteur prononçant ce jugement se positionne dans un autre camp (il n'est pas européen) et qu'il ne partage pas le point de vue qu'il critique.

Mais si nous / vous / eux ID remplissent leur mission de désignation d'un groupe ou d'une classe d'appartenance du locuteur / allocutaire, grâce, justement, à ID, se pose la question du rôle de autres auprès de ces pronoms : autres est-il redondant? Autrement dit, jusqu'à quel point les formes nous / vous / eux ID et nous autres / vous autres / eux ID sont-elles concurrentes ? Notre hypothèse est que nous ID (si l'on prend comme exemple le pronom pluriel de première personne) peut développer un sens proche de celui de nous autres $I D$ grâce à l'apposition identitaire, mais que le sens de nous tonique est différent de celui de nous autres, parce que le premier n'implique pas par lui-même une identité de classe, mais de groupe défini contextuellement délimité, alors que le deuxième implique toujours une identité catégorielle, même si ID =, , et cela par sa forme, grâce à l'ajout de autres.

Pour le montrer, remarquons d'abord que nous / vous / eux ID des énoncés de (29) à (33) acceptent l'ajout de autres, sans changement de sens: nous autres, les hommes, avons du mal à admettre que $P$; nous autres, les communistes, nous savons que $P$; vous autres, les Européens, vous êtes malades de théorie; nous autres, hommes politiques africains, devons $V$; eux autres, les Mongoles $V$. En revanche, l'équivalence ne fonctionne pas systématiquement dans l'autre sens, c'est-à-dire que les Pron. + autres n'acceptent pas la suppression de autres dans tous les cas. La suppression est difficile :

i) si le pronom disjoint est en tête de phrase et ID est un N sans déterminant non modifié :

34) Vous autres, nobles, la chasse vous perdra! ?Vous, nobles, la chasse vous perdra!

35) Il n'a pas dit un mot de toute la visite, s'écrie M. Molinier en proie à la plus fervente admiration. Nous autres, guides, sommes perplexes devant un tel enthousiasme. (Salvayre L., La Puissance des mouches, 1995, p. 87)

?Nous, guides, sommes perplexes devant un tel enthousiasme.

(ii) si nous autres / vous autres ID occupent, par exemple, dans un syntagme prépositionnel, la position de modifieur nominal :

36) La grande supériorité de vous autres dans l'enseignement sur nous autres dans les chemins de fer, c'est que lorsque vous vous salissez les mains, c'est avec du blanc de tableau, tandis que nous autres, c'est avec du noir de charbon!

? La grande supériorité de vous dans l'enseignement sur nous dans les chemins de fer, c'est que $P$.

De plus, on peut comparer avec profit les autres et autres en association avec les pronoms accentués, à savoir la possibilité de dire nous et les autres et l'impossibilité de dire *nous, les autres, $+P$ / *vous, les autres, $+P^{12}$. On comprend pourquoi : les autres anaphorique est corrélatif, c'est-à-dire qu'il réfère à un groupe se rapportant à un « repère » dont la mention précède le calcul des « individus restants » après son identification, dans les termes de Van Peteghem $\left(2000^{13}\right)$. Le pronom les autres est alors contradictoire 
avec nous accentué, qui apparaît en premier et qui deviendrait, a priori et avant toute autre information, le « repère » (comme dans nous et les autres). Cela veut dire que nous autres n'a pas de rapport sémantique avec le pronom les autres ni avec l'adjectif autres dans son emploi antéposé au nom, qui suppose 'une autre entité » dont l'altérité est calculée par rapport à un " repère » initial de même nature (un livre vs un autre livre), mais avec l'adjectif autre ${ }^{14}$ dans son emploi postposé (en position d'épithète ou d'attribut) décrit par Van Peteghem (2000 : 182) comme « relevant de l'expression d'une non-identité, non pas entre des référents mais entre des propriétés ", en somme comme une non-identité de genre. On voit la différence de sens entre autre antéposé et autre postposé : le premier présuppose, comme le font remarquer Berrendonner \& Reichler-Béguelin (1996) un 'objet-repère' de même catégorie (ayant, dans les termes des auteurs, le même 'posé préalable', désigné par le substantif du descripteur nominal ou par son hypéronyme) que le référent du descripteur nominal contenant autre, les deux termes étant «ontologiquement discernables ». Autre postposé au nom ou en position attributive ne signifie pas un contraste entre individus désignés ou désignables par le même nom, comme dans :

37) Je prends un livre, il prend un autre livre et on passe la soirée en lisant.

mais comme un contraste de propriétés qui peuvent distinguer deux états caractérisant le même individu ou des propriétés qualitatives différentes qui distinguent deux individus, comme dans :

38) Je veux un livre autre

signifiant d'une autre nature, policier et pas historique, par exemple, ou encore comme dans :

39) Paul est autre depuis la naissance de son fils (= Paul a changé)

40) A chaque saison, ce paysage est autre (= il change d'aspect).

Ces deux interprétations se vérifient si l'on compare les sens de l'adjectif autre en emploi antéposé et postposé au même $\mathrm{N}$ :

41) C'est une autre façon de parler

se rapporte corrélativement à une première façon de faire mentionnée, qui devient le 'repère' de autre $N$, parce que autre commute avec nouvelle ou deuxième :

42) C'est une nouvelle / deuxième façon de parler

mais ne commute pas avec différente :

43) *C'est une différente façon de parler.

En revanche :

44) C'est une façon autre de parler.

se comprend d'emblée comme une façon différente qualitativement, qui est d'une nature ou d'un genre différents de ceux du 'repère', comme le prouve la commutation :

45) C'est une façon différente de parler.

On peut aussi remarquer que l'on peut dire une autre fois, i.e. une seconde fois dans un enchaînement temporel mais non pas *une fois autre. Cela laisse entendre que autre antéposé réfère à une différence qui a son origine dans le positionnement local ou temporel, dans une succession, alors que postposé au nom, il réfère à une différence de nature ou de propriétés.

L'élément autres des pronoms nous autres / vous autres / eux autres est donc plus proche du deuxième emploi de autre. Tout comme autres postposé à un nom, autres postposé aux pronoms accentués produit une interprétation catégorielle et introduit une catégorie : nous sommes autres $=$ nous sommes d'un autre genre. Les propriétés de l'adjectif comme élément des Pron.+autres sont les suivantes : (i) il emprunte le nombre pluriel des pronoms disjoints signifiant des ensembles; (ii) il est postposé, favorisant l'interprétation 'différence de propriété(s); (iii) l'ensemble désigné par Pron.+autres est interprétable comme un ensemble ayant des propriétés différentes de celles d'un autre ensemble. En bref, autres 
encode l'idée d'altérité (qualité de ce qui est autre, définition de TLFi), donc de différence qualitative. Ce n'est pas l'adjectif autres qui catégorise, la catégorisation sera apportée par ID (le nom générique ou le groupe nominal postposés ou apposés aux Pron.+autres), mais il déclenche un processus anaphorique qui signifie 'groupe différent par ses propriétés', classe ou catégorie dont on met en avant la différence (signifiée par autres) qui ressort de son identité (signifiée par ID). Et pour justifier l'association Identité / Altérité, nous empruntons à Maine de Biran, par l'intermédiaire du TLFi, un argument psychologicophilosophique : «Que l'on imagine quelque changement dans la forme ou la position de l'objet connu : en glissant sur lui avec sa rapidité ordinaire, le sens percevra tout de suite le changement, et s'arrêtera dans sa course ; mais le rapport d'altérité suppose bien celui d'identité perçu auparavant. (Maine de Biran, De l'Influence de l'habitude sur la faculté de penser, 1803, p. 93. », in TLFi).

La présence de l'adjectif autres a donc son importance : avec son sens lié à la position postposée au pronom personnel pluriel, autres rend explicite l'idée de l'altérité comprise comme une différence catégorielle fondée sur une identité vue comme somme de propriétés. Sans une apposition identitaire, toute possibilité d'altérité catégorielle est ouverte, d'où l'utilisation de nous autres seul comme titre de roman ou comme intitulé d'émission de radio, d'où aussi la possibilité d'avoir un $\mathrm{N}$ générique sans déterminant comme apposition, dont le rôle est d'expliciter l'idée de catégorie introduite par autres. Le pronom catégoriel nous autres réunit identité et altérité, dans le sens où l'altérité provient d'une identité sur laquelle elle se fonde et sans laquelle elle n'existerait pas. La forme nous autres isolée de tout contexte amène à chercher à quoi correspond cette altérité, à se questionner sur le statut de la classe à laquelle appartient l'énonciateur et sur ses propriétés (en quoi sont-ils autres?).

En bref, les formes nous / vous / eux ID ont un emploi catégoriel (et contrastif) grâce à ID, alors que nous autres / vous autres / eux autres sont explicitement catégoriels (et potentiellement contrastifs) et peuvent se dispenser de ID explicite.

\section{Prédications et effets pragmatiques}

\subsection{Affirmation identitaire}

L'idée d'identité - altérité de la classe d'appartenance de l'énonciateur de nous autres ne dépend pas, comme nous l'avons déjà dit, de la prédication, mais ouvre la voie à tout prédicat qui est conforme avec l'idée stéréotypée que l'on se fait de la classe ID. C'est pour cette raison que les pronoms contrastifs peuvent s'utiliser pour l'affirmation identitaire de la classe d'appartenance du locuteur, avec une mise en second plan de l'idée de contraste, comme l'illustrent les exemples suivants :

46) À Genève, en 1954, nous avions solennellement, nous autres Français, avec l'appui des Soviétiques, des Chinois et des Britanniques, promis au peuple vietnamien qu'il pourrait librement déterminer son évolution ... (Mendès-France P., Euvres complètes, 1989, p. 561)

47) [...] elle était éblouie par ses trouvailles de conteur, ses brusques envolées prophétiques et troublée par ces mystérieux souvenirs imaginaires... Mais étaient-ils imaginaires ? Quand il s'arrêtait, elle remettait en marche cette étrange machine à paroles. - Tu dis toujours : "Nous autres les druides ", mais les druides sont à la première page de notre histoire de France, et nous, nous sommes à la dernière?... - C'est parce que ce que vous appelez l'histoire de France est trop court pour moi! (Vincenot H., Le Pape des escargots, 1972, p. 306)

48) Devant cette affirmation publique, massive, d'une identité supposée commune - "Nous autres psychanalystes, experts assermentés de l'Inconscient "-, je m'éclipse. (Pontalis J.-B., Fenêtres, 2000, p. 18)

49) Des probités comme Monsieur Necker, il faudrait les pousser vers les sommets ! "Le nouveau Colbert", voilà comment nous l'avons baptisé, nous autres économistes, à Guéret ! (Chandernagor F., L'Enfant des Lumières, 1995, p. 132-133) 
C'est le cas aussi de deux classes concernées par la même information rhématique, comme l'analogie entre la classe les animaux sauvages et la classe les hommes qui se sentent en sécurité dans leurs territoires respectifs (opposition ontologique, mais ressemblance dans un comportement renseigné par le contenu prédicatif) ou comme l'affirmation pure et simple de la ressemblance selon certains critères entre deux peuples :

50) La plupart des animaux sauvages ont un territoire à eux. C'est à l'intérieur de celui-ci que se déroule leur vie, et ils n'aiment guère à en franchir les limites. Nous autres aussi, êtres humains avons - me semble-t-il - notre territoire, et dès que nous le quittons, nous perdons ce sentiment de sécurité que nous en recevions. (Juliet C., Accueils. Journal IV, 1982-1988, 1994, p. 246-247)

51) Il me semble enfin que vous autres, Argentins, ressemblez à nous, Français, quant à vos conceptions et à vos intentions relatives au monde où nous sommes. (De Gaulle C., Discours et messages. 4. Pour l'effort. 1962-1965, 1970, p. 290)

L'affirmation identitaire permet de dire que l'identité est première et que le contraste entre deux classes ou groupes découle de celle-ci; elle est donnée par le sens des mots en intension, mais se compose surtout de toutes les connotations culturelles ou stéréotypiques, positives ou négatives, qui l'accompagnent. Ou, comme le souligne Kleiber (2012: 151-152 et 155), la spécificité sémiotique des pronoms (et de toutes les expressions token-réflexives, qui aident à trouver leurs référents indexicalement, c'est-à-dire par l'intermédiaire d'éléments spatio-temporellement reliés à leur occurrence) réside dans le fait que je et $t u$ sont « des marques linguistiques permettant à un locuteur d'exprimer ou de représenter la conscience qu'il a de soi-même, de celui auquel il parle (donc de son interlocuteur) », ce qui explique une propriété reconnue depuis les études de Benveniste sur les pronoms : «Je et $t u$ apparaissent ainsi comme des marqueurs de subjectivité, dimension qui ouvre directement sur d'autres champs disciplinaires comme la psychologie, la psychanalyse, la philosophie, la sociologie, la littérature, la religion, etc. (Kleiber, 2012 : 155). Comme je / moi et tu / toi sont sémantiquement compris dans nous et vous, les formes nous autres / vous autres / eux autres permettent à un locuteur d'exprimer la conscience qu'il a de sa classe d'appartenance (et, par cela, de soi même du point de vue de l'une identité de classe), mais aussi la représentation qu'il se fait d'autres catégories ayant le trait [+humain], dont il se sent ontologiquement différent.

\subsection{Contraste identitaire et contraste prédicatif}

L'identité - altérité signifiée par les pronoms catégoriels s'accorde naturellement avec une prédication contrastive ou un autre type de contenu propositionnel signifiant un contraste, comme le montrent les exemples suivants :

52) D’ailleurs, les séjours parisiens étaient marqués d'une amertume que les récits de ma grandmère ne parvenaient pas à dissimuler. Quelque dissension familiale dont il ne nous était pas donné de connaître les raisons? Ou bien une froideur très européenne dans les relations entre les proches, inconcevable pour nous autres, Russes, avec notre collectivisme débordant? (Makine A., Le Testament français, 1995, p. 61)

53) Je crois que le censeur de la Préfecture a dî se dire en sortant : Les Russes ignorent l'eau de rose, il faudra que nous leur en fournissions, nous autres Français, spécialistes en la matière. (Aragon L., Euvre poétique : tome 1 : livre 3 (1926), 1982, p. 938)

54) Nous sommes pour le communisme avant d'être contre Staline. Ce sont des choses que vous autres, démocrates de l'Ouest, libéraux de tout poil, vous avez, j'imagine, un peu de mal à comprendre. (d'Ormesson J., Le Bonheur à San Miniato, 1987 pp. 24-25)

L'expression du contraste peut émaner de la classe ID ou lui être attribuée, parce qu'elle renvoie à des propriétés qui lui correspondent, qui la définissent, dans lesquelles la classe ID se reconnaît, ou que l'on démasque, qui la mettent en contraste avec une autre, définitoirement opposée ( $c f$. froideur européenne vs 
collectivisme russe débordant; connaisseurs des parfums vs ignorant les parfums; comprenant le communisme vs ne comprenant pas le communisme). Cet aspect méritera d'être développé, parce que les prédications contrastives ouvrent la voie à une interprétation pragmatique des actes de langage indirectement effectués. L'énonciateur choisit de dire nous et pas moi dans une stratégie de communication par laquelle il s'attribue le statut de porte-parole de la classe d'appartenance, avec des effets pragmatiques divers ${ }^{15}$ : solidarité de classe, souhait d'affirmer les différences, tentative de disculper une faute individuelle, accusation, effacement de l'individualité derrière l'autorité d'une classe, etc. ${ }^{16} \mathrm{Si}$ l'énoncé comprend la forme eux autres ID (et même eux, les $N$ ), il a une dimension polyphonique ou correspond à un discours rapporté, parce que la présence de ces pronoms dans l'énoncé suppose un point de vue subjectif à l'égard des leurs référents ou de la classe qu'ils visent (eux autres, les malheureux $+\mathrm{P}$; eux, les Mongols $+\mathrm{P})$. L'émetteur du point de vue subjectif et repérable grâce à l'idée de mise en contraste qui suppose un deuxième terme de la relation oppositive.

\subsection{Appropriation individuelle d'un jugement générique}

Le fait le plus marquant qui découle de l'affirmation par l'énonciateur de l'appartenance à une classe est l'appropriation individuelle d'un jugement générique. Nous avons anticipé cette idée en montrant que, par leur sens et par leur référence, nous autres ID et vous autres ID réunissent énonciation et catégorisation. C'est pour cette raison que les énoncés contenant ce type de pronoms, qui impliquent un eux générique, peuvent être reformulés en phrases génériques standard, qui peuvent être envisagées sans rapport avec une situation d'énonciation. C'est-à-dire que la suppression des pronoms catégoriels et des pronoms conjoints correspondants et donc l'effacement de toute marque d'énonciation ont comme résultat l'obtention de phrases génériques :

55) a. Nous autres policiers, nous ne sommes que des auxiliaires de la justice. (Boudard A., Mourir d'enfance, 1995, p. 181)

b. Les policiers ne sont que des auxiliaires de la justice.

56) a. Nous autres, les acteurs, ne sommes pas comme les musiciens. Nous sommes obligés, quand nous jouons, de rester sur la scène pendant toute la durée d'une scène. (Roubaud J., Nous, les moins-que-rien, Fils aînés de personne, 12 (+1) autobiographies, 2006, p. 84)

b. Les acteurs ne sont pas comme les musiciens. Ils sont obligés, quand ils jouent, de rester sur la scène pendant toute la durée d'une scène.

57) a. Rien n'est plus évident, pour nous autres, les hommes, que ce monde où nous vivons. (d'Ormesson J., La Douane de mer, 1993, p. 39)

b. Rien n'est plus évident pour les hommes que le monde où ils vivent.

58) a. Ce que vous êtes bêtes, vous autres, les garçons, me dit Elena, vous ne remarquez jamais rien! (Cendrars B., Bourlinguer, 1948, p. 130)

b. Les garçons sont bêtes, ils ne remarquent jamais rien.

Certaines phrases génériques obtenues peuvent être proches des phrases analytiques, comme dans (55b), faisant part d'un jugement générique d'un type particulier ( $c f$. l'insertion de la restriction dans la phrase attributive), parce qu'il correspond à l'image que se fait un certain locuteur des catégories du monde humain et de la société, image qui est, à la fois, valable pour l'énonciateur et potentiellement contestable pour d'autres, ce qui les rapproche des phrases génériques typifiantes locales ( $c f$. sur les phrases génériques analytiques, typifiantes et typifiantes locales, Kleiber, 1978 et 1988 et Anscombre, 1995). Ce sont des jugements qui correspondent plus à des stéréotypes culturels et à un phénomène de connotation lexicale qu'à une définition analytique du monde. L'utilisation des pronoms contrastifs nous autres / vous autres $I D$ entre deixis et catégorisation se complète donc par cet emploi qui réunit deixis et généricité au niveau de l'énoncé. Cet emploi concorde avec l'affirmation de la subjectivité, de l'appartenance, en tant qu'énonciateur, à une catégorie du monde humain, relationnel, empreint de subjectivité. 


\section{Conclusion}

Pour finir, on peut distinguer dans l'emploi des pronoms personnels pluriels [+humain] les cinq cas suivants :

a. Vous aimez les feignants.

b. Vous, vous aimez les feignants.

c. Vous, les femmes, vous aimez les feignants.

d. Vous autres, les femmes, vous aimez les feignants.

e. Vous autres, vous aimez les feignants.

Le premier, (a), correspond au pronom personnel conjoint au verbe. Le deuxième, (b), correspond au pronom personnel disjoint, que nous avons appelé 'simple' le long de cette analyse, pour le distinguer de la forme composée avec l'adjectif. La forme disjointe crée un effet contrastif, qui provient du pronom tonique lui-même et qui est explicité par le prédicat bornant un groupe spécifique (vous qui aimez les feignants) et laissant entendre que d'autres variables (moi, lui, eux) ne vérifient pas ce prédicat. Le troisième cas, (c), illustre l'emploi catégoriel (réunissant énonciation et généricité) du pronom accentué, qui est dû à la présence de l'apposition identitaire, en l'absence de laquelle l'énoncé serait assimilable au cas (b) du contraste accentué prédicatif ${ }^{17}$. Le quatrième et le cinquième cas, (d) et (e), sont ceux du pronom composé catégoriel (réunissant énonciation et généricité), le pronom de l'identité - altérité explicite, grâce à autres, avec une double possibilité : l'expression de l'identité de classe ou son inférence.

Autrement dit, pour les pronoms disjoints, qui intéressent cette analyse, on a distingué les situations suivantes :

- nous / vous / eux disjoints contrastifs contextuels, signifiant un contraste de groupe contextuellement délimité, dépendant de la prédication, comme dans nous, nous avons vu le film, mais lui, il ne l'a pas vu;

- nous / vous / eux ID en emploi tonique contrastif catégoriel grâce à une apposition identitaire explicite, comme dans nous, les Français, $P$;

- nous autres / vous autres (ID) toniques catégoriels signifiant explicitement une identité altérité catégorielle, comme dans nous autres Français ou nous autres, les Français.

Le fait que les Pron.+autres n'ont que des formes de pluriel se comprend maintenant : le pluriel n'est pas lié à l'idée de contraste, mais à l'idée de classe naturelle concrète [+humains], qui suppose une multitude d'éléments discrets, à l'emploi catégoriel [+humain] pour lequel ils sont spécialisés.

Le contraste rattaché à nous autres / vous autres ID catégoriels bi-référentiels provient de l'affirmation de l'altérité qui implique une identité de classe (donc générique), comprise comme une somme de propriétés dénotatives mais aussi connotatives, culturelles et stéréotypiques ; eux autres (ID) non énonciatif est en fait un nous autres ou vous autres en discours indirect. Il reste à approfondir les processus anaphoriques qui régissent l'emploi des Pron.+ autres, ou encore, dans un autre domaine, les actes pragmatiques accomplis par leur utilisation. La stratégie de l'énonciateur comme porte-parole d'une classe permet sûrement de présenter une vision du monde, des jugements quasi stéréotypés, des opinions en rapport avec l'identité du groupe auquel appartient ou auquel s'oppose l'énonciateur en tant qu'individu. C'est une manière de signifier comment le sujet énonciateur s'approprie et utilise l'idée de classe ou de catégorie correspondant aux humains, à son monde d'existence et de croyance. 


\section{Références bibliographiques}

Anscombre, J.C. (1995). Théorie des topoï. Paris : Kimé.

ATILF, CNRS, Université Nancy 2. 2004, mise à jour 2008. Base textuelle Frantext, http://www.frantext.fr.

Benveniste, E. (1966). Problèmes de linguistique générale, Paris : Gallimard.

Berrendonner, A. \& Reichler-Béguelin, M.-J. (1996). « De quelques adjectifs à rendement anaphorique : premier, dernier, autre ». Studi italiani di linguistica teorica e applicata, XXV, 3, 475-502.

Bonnard, H. (1950 / éd. 1997). Grammaire française à l'usage de tous. Paris : Magnard.

Grevisse, M. (2006 / 13 édition par Goosse, A.). Le Bon Usage, Paris / Louvain-la-Neuve : DeBoeckDuculot.

Kerbrat-Orecchioni, C. (1999). L'énonciation. De la subjectivité dans le langage, $4^{\mathrm{e}}$ édition. Paris : Armand Colin.

Kerbrat-Orecchioni, C. (2001). Les actes de langage dans le discours. Théorie et fonctionnement. Paris. Armand Colin.

Kleiber, G. (1978). Phrases et valeurs de vérité. In Martin, R. (éd). La notion de recevabilité en linguistique, Paris : Klincksieck, 21-65.

Kleiber, G. (1981). Problème de référence. Descriptions définies et noms propres. Paris : Klincksieck.

Kleiber, G. (1986). Déictiques, embrayeurs, «token-reflexives », symboles indexicaux : comment les définir ?. L'information grammaticale, 30, 3-22.

Kleiber, G. (1988). Phrases génériques et raisonnement par défaut. Le français moderne, 56, $n^{\circ}$ 1-2, 1-15.

Kleiber, G. (1994). Anaphores et pronom. Louvain-la-Neuve : Duculot.

Kleiber G. (à par. 2012), A la quête de Je et Tu. In Frath, P., Blomfield, J., Bourdier V., Bréhaux, K., Hilgert, E. (éds). La référence, la conscience et le sujet énonciateur. Reims : EPURE, 135-162.

Lammert M. (2010), Sémantique et cognition : les noms collectifs. Genève : Librairie Droz.

Maingueneau, D. (1994 / édition 1999). L'Enonciation en linguistique française. Paris : Hachette.

Moignet, G. (1981). Systématique de la langue française. Paris : Klincksieck.

Palma, S. (à par. 2012), Sujet énonciateur entre deixis et catégorisation : le cas de nosotros / vosotros (esp.) », in Frath, P., Blomfield, J., Bourdier V., Bréhaux, K., Hilgert, E. (éds). La référence, la conscience et le sujet énonciateur. Reims : EPURE.

Pic, E. \& Furmaniak, G. (à par. 2012). Le degré de spécialisation comme facteur de variation de la représentation du JE. In Frath, P., Blomfield, J., Bourdier V., Bréhaux, K., Hilgert, E. (éds). La référence, la conscience et le sujet énonciateur. Reims : EPURE.

Pottier, B. (2002). Autour de nous. In Carel, M. (éd.). Les facettes du dire, Paris : Kimé. 237-240.

Rey, A. (dir.). (2006). Dictionnaire historique de la langue française. Paris : Le Robert.

Riegel, M., Pellat J.-C. \& Rioul R. (1994 / 9 éd. 2009). Grammaire méthodique du français. Paris : P.U.F.

Ronat, M. (1979). Pronoms topiques et pronoms distinctifs. Langue française, 44, 106-128.

Rosier, L. (2006). Les locutions « Nous autres » et « Nous autres + (X) »: mais de quels « autres » s'agitil? In Kleiber, G., Schnedecker, C., Theissen, A. (éds). La relation partie - tout. Louvain Paris : Editions Peeters, 153-166.

Schapira, C. (2010) Nous et eux. Les nationalismes et leur expression dans les media. Paris: L'improviste. 179-191.

Tamba, I. (1994). Pronominaux personnels en français et en japonais. Faits de langues, 3. 221-224.

Van Peteghem, M. (1995). L'"indéfini" AUTRE : analyse sémantico-référentielle ou en quoi autre est déictique. In van Deyck R. (éd.). Diachronie et variation linguistique. La déixis temporelle, spatiale et personnelle. Gand : Communication \& Cognition, 87-114.

Van Peteghem, M. (1997). Mécanismes anaphoriques sous-jacents aux « indéfinis » autre et même. In De Mulder, W., Tasmowski-De Ryck, L. et Vetters C. (éds). Relations anaphoriques et (in) cohérence. Amsterdam / Atlanta : Rodopi, 187-200.

Van Peteghem, M. (2000). Les indéfinis corrélatifs autre, même et tel. In Bosveld-de Smet, L., Van Peteghem, M. et Van De Velde, D. (éds). De l'indétermination à la qualification. Les indéfinis. Arras : Artois Presses Université, 117-202. 
Van Peteghem, M. (2001a). Autre et même sans nom : anaphore nominale ou pronominale ?. In De Mulder W., Vet C. \& Vetters C. (éds). Anaphores pronominales et nominales. Etudes pragmasémantiques. Amsterdam / New York : Rodopi, 123-143.

Van Peteghem, M. (2001b). Autre vs différent : du pareil au même ?. In Amiot, D., De Mulder, W. et Flaux, N. (éds). Le syntagme nominal : syntaxe et sémantique. Arras: Artois Presses Université, 141-160.

\begin{abstract}
1 Article publié également courant 2012, en version courte, sous le titre « Sujet énonciateur entre deixis et catégorisation: le cas de nous autres / vous autres (fr.) », in Frath, P., Blomfield, J., Bourdier V., Bréhaux, K., Hilgert, E. (éds). La référence, la conscience et le sujet énonciateur. Reims : EPURE. Les ajouts à la version présente sont la suite des remarques des relecteurs et rapporteurs du CMLF2012, que je remercie.
\end{abstract}

${ }^{2}$ Cf. l'exemple de Kleiber (1994 : 137) Paul est parti. LUI aurait pu me secourir qui laisse entendre que «d'autres n'auraient pas pu me secourir ». Cf. aussi à cet égard Ronat (1979) et Pottier (2002).

${ }^{3} \mathrm{Du}$ point de vue sémantique, c'est Pron.-même (moi-même, nous-mêmes, etc.) que l'on peut considérer comme un pronom renforcé.

${ }^{4}$ Une recherche dans Frantext en mars 2012 a donné une liste de 53 occurrences de eux autres.

${ }^{5}$ L'opinion sur l'emploi uniquement des $1^{\text {ère }}$ et $2^{\mathrm{e}}$ personnes est quasi générale, $c f$. à cet égard Rosier (2006) ou la GMF (2009).

${ }^{6}$ L'emploi de vous autre au singulier n'a été trouvé que dans la correspondance de Sartre à Beauvoir ( $c f$. Frantext et Grevisse / Goosse, 2006). Mais ce n'est pas la seule forme originale née de la plume du philosophe : il s'adresse, par exemple, au « Castor » en parlant aussi de nous autres deux (cf. Frantext).

${ }^{7}$ Nous nous proposons une étude de fréquence de ces différentes formes dans la base textuelle Frantext.

8 Pour Moignet (1981: 160), les formes accentuées (ou «pronoms prédicativés ») présentent les personnes « comme des êtres en soi, indépendamment des phénomènes de l'existence ».

9 Je / moi et tu / toi sont, comme le montre Kleiber (1986 et 2012 : 151-152), des expressions tokenréflexives, c'est-à-dire des expressions indexicales, "parce qu'elles obligent à trouver le référent par le truchement de leurs occurrences. Comme une occurrence ou instance d'une expression linguistique est unique et se trouve délimitée soit par le moment de son énonciation, s'il s'agit d'une occurrence sonore, soit par le lieu où elle est inscrite, s'il s'agit d'une occurrence écrite, s'il faut partir de cette occurrence pour trouver le référent visé, alors ce ne sont que des éléments spatio-temporellement contigus à l'occurrence qui permettent d'accéder à ce référent. ».

${ }^{10}$ Cf. la présentation du roman Nous autres, les Sanchez (1961) de Catherine Paysan, sur le site Livrenpoche.com: "En Normandie, les Mexicains ne courent pas les rues. Amaryllis Monceau, l'institutrice de St-Christophe a découvert le sien à Paris. Sanchez est peintre, elle est belle. Trois semaines d'une cour ardente. Ils se marient. Ainsi fut fondée dans les années 30, la famille Sanchez. L'homme a continué à peindre, la femme à enseigner. Trois enfants leur sont nés. C'est en voyant les gens se retourner sur leur groupe insolite, que Carlos, le fils aîné, prend conscience de la singularité de son clan, au milieu des paysans vikings. ") (http://www.livrenpoche.com/livre/Nous-autres-lesSanchez/24325.html, 15 mars 2012)

11 Cf. à cet égard l'interprétation <COMMUNAUTE> de we / nous que Pic \& Furmaniak (2012) décrivent comme «la tendance de l'auteur à s'assimiler à la classe des experts » et comme une sousclasse de we / nous < GENERIQUE > signifiant « l'humanité ».

${ }^{12}$ La base textuelle Frantext fait part d'un hapax pour chaque forme.

13 Cf. aussi, sur autre «dans tous ces états », Van Peteghem (1995, 1997, 2001a et 2001b), ou Berrendonner, A. \& Reichler-Béguelin (1996) pour les mécanismes anaphoriques à l'œuvre dans le cas de autres antéposé au nom.

${ }^{14}$ Point de vue défendu aussi par Rosier (2006).

${ }^{15} C f$., pour les actes de langage indirects, Kerbrat-Orecchioni (2001).

${ }^{16} C f$. Schapira (2010) sur un emploi pragmatique de nous et eux « communautaires » ou « nationalistes ».

${ }^{17}$ Dans des langues qui n'ont pas de pronoms marqués « catégoriels », comme l'anglais ou l'espagnol, le pronom personnel simple peut avoir une interprétation catégorisante en contexte, $c f$. à cet égard Palma (2012) et Pic \& Furmaniak (2012). 